\title{
OVERALL MODULI OF HETEROGENEOUS ELASTIC MATERIALS
}

\section{Muneo HORI* and Takashi MIURA**}

\begin{abstract}
The estimate of overall behaviors of heterogeneous or damaged materials has been one of major concerns for engineers of various fields. From a micromechanical point of view, identified are fundamental relations among the average field quantities from which overall responses of the materials can be determined. A new estimate method called the finite part approximation is proposed which may overcome deficiencies of existing estimate methods. A solid damaged by microcracks is considered as an illustrative example, and the overall moduli estimated by the proposed method are compared with those predicted by the existing ones.
\end{abstract}

Keywords: overall monduli, heterogeneity, averaging scheme

\section{INTRODUCTION}

Estimate of overall or effective properties of heterogeneous materials, such as composites, concretes, rocks, or damaged solids, has been investigated by a number of researchers ; see Christensen $^{1)}$ and Hashin $^{2)}$ for literatures, and reference $^{3)}$ for civil engineering materials. Although these materials consist of sets of inhomogeneities in a micro-scopic length scale, they can be regarded as homogeneous if viewed in a macro-scopic length scale sufficiently larger, say, $10^{2}$ or $10^{3}$ times, than microconstituents ; see Fig. 1 and $\mathrm{Hill}^{4)}$. Hence, the overall material properties can be determined by relations among the macroscopic behaviors which are represented by average responses of microconstituents. In particular, if all microconstituents are linearly elastic, the overall (elastic) moduli can be defined.

From this physical observation, it is seen that a reasonable estimate of the overall moduli requires to analyze responses of each microconstituent and to take volume average of them. Two kinds of mathematical models for this purpose have been studied, a representative volume element (RVE) model and a periodic structure (PS) model. They are summarized as follows:an RVE model considers a finite body with statistically random microstructures, and estimates the overall moduli in a simple analytical expression with the aid of exact solutions of elasticity ; on the other hand, a PS model deals with a material with a periodic microstructure, and computes the overall moduli

\footnotetext{
* Member of JSCE, Ph.D., Lecturer, Dept. of Civil Eng., Tohoku Univ. (Aoba, Sendai)

** Member of JSCE, Dr. Eng., Professor, Dept. of Civil Eng., Tohoku Univ.
}

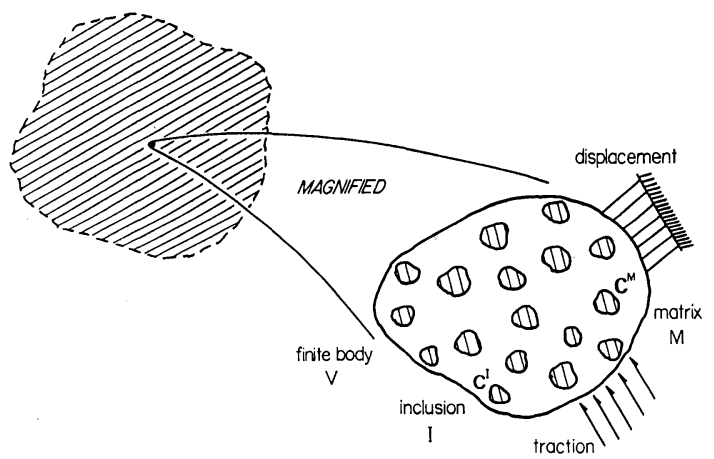

Fig.1 Heterogeneous material and finite body.

using Fourier expansion, which requires tedious numerical computational efforts.

In this paper, we consider averaging schemes used in RVE modeling, which can be divided into the following two categories:the one to estimate exact values of the overall moduli and the other to estimate bounds for the over moduli. The former includes, for example, the law of mixture, the dilute distribution assumption, or the self-consistent method; see Nemat-Nasser and Hori ${ }^{5)}$. The latter is represented by the Hashin-Shtrikman variational principle; see Willis ${ }^{(,), 7)}$. Although there are plenty of minor modifications for these averaging schemes, typical formulations are considered.

Recently, Benveniste ${ }^{8)}$ proposed a new averaging scheme for composites, and Mori and Wakashima ${ }^{9}$ found a similar averaging scheme. It appears that these two methods are based on a more reasonable concept of averaging than conventional ones. We generalize this concept to establish systematical foundations for a new averaging scheme which might cover the existing averaging schemes. To this 
end, first, basic exact relations among the average field quantities are summarized in Sct. 2. Three conventional averaging schemes are derived from them in Sct. 3. Introducing the finite part concept, from which the averaging scheme of Benveniste or Mori and Tanaka can be derived in a transparent manner, we formulate a new averaging scheme in Sct. 4, and compare it with the existing ones in Sct. 5. Finally, in Sct. 6, a solid damaged by microcracks is considered as a simple example to demonstrate the effectiveness of the proposed averaging scheme.

\section{AVERAGE FIELD QUANTITIES OF FINITE BODY}

In this section, we derive exact volume averages of physical field variables in a finite body $V$ (There have been found considerable misunderstandings on them ; serious mistakes were made by even Christensen $^{1)}$ and Hashin $^{2)}$ ). Three-dimensional Cartesian coordinate systems $x_{i}(i=1,2,3)$ are taken in $V$. Strain, stress, and displacement fields are denoted by $\varepsilon=\varepsilon(x), \sigma=\sigma(x)$, and $\boldsymbol{u}=\boldsymbol{u}(\boldsymbol{x})$. For brief expressions, a tensor is written in a bold letter, and the first-, second-order contractions, and tensor product are denoted by $\cdot$, : and $\otimes$, respectively. Assuming small deformation, static equilibrium, and linear elasticity, we have

$$
\begin{aligned}
& \varepsilon=\frac{1}{2}\left\{\nabla \otimes \boldsymbol{u}+(\nabla \otimes \boldsymbol{u})^{T}\right\}, \quad \nabla \cdot \boldsymbol{\sigma}=0, \quad \boldsymbol{\sigma}=\boldsymbol{C}^{\prime}: \varepsilon, \\
& e=\frac{1}{2} \varepsilon: \boldsymbol{C}^{\prime}: \varepsilon\left(=\frac{1}{2} \boldsymbol{\sigma}: \varepsilon\right) \cdots \cdots \cdots \cdots \cdots \cdots(1-\mathrm{a} \sim \mathrm{d})
\end{aligned}
$$

where $\boldsymbol{C}^{\prime}=\boldsymbol{C}^{\prime}(\boldsymbol{x})$ and $e=e\left(\varepsilon, \boldsymbol{C}^{\prime}\right)$ are heterogeneous elasticity of $V$ and (elastic) strain energy density, and $\nabla$ is defined by $(\nabla)_{i} \equiv \partial / \partial x_{i}$.

The volume average of $\varepsilon, \sigma$, and $e$ is derived from (1) with the use of Gauss theorem. Denoting the volume average taken over $V$ by $\langle\cdots\rangle_{V}$, we have

$$
\begin{aligned}
& \langle\varepsilon\rangle_{V}=\frac{1}{V} \int_{\partial V} \frac{1}{2}(\boldsymbol{\nu} \otimes \boldsymbol{u}+\boldsymbol{u} \otimes \nu) d s, \\
& \langle\boldsymbol{\sigma}\rangle_{V}=\frac{1}{V} \int_{\partial V} \boldsymbol{t} \otimes \boldsymbol{x} d s, \\
& \langle\boldsymbol{e}\rangle_{V}=\frac{1}{V} \int_{\partial V} \frac{1}{2} \boldsymbol{t} \cdot \boldsymbol{u} d s
\end{aligned}
$$

where $\nu$ is the unit normal on the boundary surface $\partial V$, and $\boldsymbol{t}=\boldsymbol{\nu} \cdot \boldsymbol{\sigma}$. The following relations among $\langle\varepsilon\rangle_{V},\langle\boldsymbol{\sigma}\rangle_{V}$, and $\langle e\rangle_{V}$ are derived from $(1 \sim 4)$ :

$$
\begin{aligned}
& \langle e\rangle_{V}-\frac{1}{2}\langle\boldsymbol{\sigma}\rangle_{:}\langle\boldsymbol{\varepsilon}\rangle_{V}=\frac{1}{V} \int_{\partial V} \frac{1}{2}\left(\boldsymbol{t}-\nu \cdot\langle\boldsymbol{\sigma}\rangle_{V}\right) \cdot \\
& \quad\left(\boldsymbol{u}-\boldsymbol{x} \cdot\langle\boldsymbol{\varepsilon}\rangle_{V}\right) d s, \\
& \frac{\partial}{\partial\langle\boldsymbol{\varepsilon}\rangle_{V}}\langle\boldsymbol{e}\rangle_{V}=\langle\boldsymbol{\sigma}\rangle_{V^{\prime}}
\end{aligned}
$$

where $\langle e\rangle_{V}$ is now assumed to be function of $\langle\varepsilon\rangle_{V}$.
From (5) and (6), it is seen that the two overall moduli, $\overline{\boldsymbol{C}}$, the ones defined through an average stress-strain relation and the others defined through an average strain energy relation, i.e.,

$$
\begin{array}{r}
\langle\boldsymbol{\sigma}\rangle_{V} \equiv \overline{\boldsymbol{C}}:\langle\boldsymbol{\varepsilon}\rangle_{V}, \quad \text { and }\langle\boldsymbol{e}\rangle_{V} \equiv \frac{1}{2}\langle\boldsymbol{\varepsilon}\rangle_{V}: \overline{\boldsymbol{C}}:\langle\boldsymbol{\varepsilon}\rangle_{V} \\
\ldots \ldots \ldots \ldots \ldots \ldots \ldots \ldots \ldots \ldots \ldots \ldots \ldots \ldots \ldots \ldots \ldots \ldots \ldots \ldots \ldots \\
\end{array}
$$

may not coincide with each other (Note that (6) guarantees that differentiation of (7-b) with respect to $\langle\varepsilon\rangle_{V}$ yields $\left.\langle\boldsymbol{\sigma}\rangle_{V}\right)$.

If the right side of (5) vanishes, (7-a) and (7-b) yield the same $\bar{C}$. There are two types of boundary conditions on $\partial V$ satisfying this condition, linear displacements, $u=x \cdot \varepsilon^{\circ}$, and uniform tractions, $t=$ $\nu \cdot \sigma^{\circ}$, with constant $\varepsilon^{\circ}$ and $\sigma^{\circ}$. It is often taken for granted that $\overline{\boldsymbol{C}}$ are uniquely determined whether the linear displacements or the uniform tractions are prescribed ; see Christensen ${ }^{1)}$ or Hashin ${ }^{2}$. However, it is indeed the case that the relations among the average field quantities depend on the surface data. To illustrate this, compute the difference of the average strain energy due to various boundary conditions. Denoting field variables due to the uniform tractions, the linear displacements, and other arbitrary general boundary conditions by putting superscripts $\Sigma, E$, and $G$, respectively, on them, we have

$$
\begin{aligned}
& \left\langle e^{G}\right\rangle_{V}-\left\langle e^{\Sigma}\right\rangle_{V}=\frac{1}{2}\left\langle\left(\varepsilon^{G}-\varepsilon^{\Sigma}\right): C^{\prime}:\left(\varepsilon^{G}-\varepsilon^{\Sigma}\right)\right\rangle_{V} \geqq 0 \\
& \text { (for } \left.\left\langle\varepsilon^{G}\right\rangle_{V}=\left\langle\varepsilon^{\Sigma}\right\rangle_{V}\right) \\
& \left\langle e^{G}\right\rangle_{V}-\left\langle e^{E}\right\rangle_{V}=\frac{1}{2}\left\langle\left(\sigma^{G}-\sigma^{E}\right): C^{\prime-1}:\right. \\
& \left.\left(\boldsymbol{\sigma}^{G}-\boldsymbol{\sigma}^{\Sigma}\right)\right\rangle_{V} \geqq 0 \quad\left(\text { for }\left\langle\boldsymbol{\sigma}^{G}\right\rangle_{V}=\left\langle\boldsymbol{\sigma}^{E}\right\rangle_{V}\right) \\
& \text {................................ }(8-\mathrm{a}, \mathrm{b})
\end{aligned}
$$

see Nemat-Nasser and $\mathrm{Hori}^{\mathrm{s}}$. Inequalities (8-a) and (8-b) show the dependence of the average responses on the boundary conditions.

\section{CONVENTIONAL AVERAGING SCHEMES}

For simplicity, we consider a two-phase material consisting of matrix and inclusion phases to present typical conventional averaging schemes ; microinclusions with common elasticity $\boldsymbol{C}^{I}$ are embedded in the matrix with elasticity $\boldsymbol{C}^{M}$. The overall moduli of this material are evaluated by average field relations for a finite body, $V$, which represents the material microstructure in a statistical sense (RVE modeling). Denoting the matrix and inclusion phases in $V$ by $M$ and $I$, we can decompose $V$ into $M+I$ and define the volume fraction of $I$ by $f \equiv$ $I / V$. Hence, the average stresses and strains over $V, M$, and $I$ must satisfy

$$
\langle\boldsymbol{\sigma}\rangle_{V}=(1-f)\langle\boldsymbol{\sigma}\rangle_{M}+f\langle\boldsymbol{\sigma}\rangle_{I},
$$




$$
\begin{aligned}
& \langle\varepsilon\rangle_{V}=(1-f)\langle\varepsilon\rangle_{M}+f\langle\varepsilon\rangle_{I} \\
& \langle\boldsymbol{\sigma}\rangle_{M}=C^{M}:\langle\varepsilon\rangle_{M},\langle\boldsymbol{\sigma}\rangle_{I}, \quad\langle\sigma\rangle_{I}=C^{I}:\langle\varepsilon\rangle_{I} \\
& \text {............................. }(9-\mathrm{a} \sim \mathrm{d})
\end{aligned}
$$

where $\langle\cdots\rangle_{M}$ and $\langle\cdots\rangle_{I}$ denote the volume average taken over $M$ and $I$. Note that unless it does not make any confusion, a letter, say, $V$, can represent region, volume, or typical geometry, depending on the context.

As is seen from $(9-a \sim d)$, only one relation among the above six average field quantities is required to estimate $\bar{C}$ defined by (7-a). An RVE modeling seeks to obtain a relation of $\langle\varepsilon\rangle_{I}$ (or $\left.\langle\boldsymbol{\sigma}\rangle_{I}\right)$ to $\langle\varepsilon\rangle_{V}\left(\right.$ or $\left.\langle\boldsymbol{\sigma}\rangle_{V}\right)$. The simplest approximation is that $\langle\varepsilon\rangle_{I}$ or $\langle\boldsymbol{\sigma}\rangle_{I}$ equals the average strain or stress over the surrounding solid. For a more sophisticated approximation, Eshelby ${ }^{10), 11)}$ obtained the following relation for an ellipsoidal inhomogeneity $\Omega$ with elasticity $C^{\Omega}$ embedded in an infinite uniform domain $B^{\infty}$ with elasticity $C$ : when $B^{\infty}$ is subjected to far-field strains $\varepsilon^{\infty}$, the strain and stress fields become constant in $\Omega$, i.e.,

$$
\varepsilon=(\boldsymbol{I}+\boldsymbol{S}: \Psi): \varepsilon^{\infty}, \boldsymbol{\sigma}=\boldsymbol{C}:(\boldsymbol{I}+(\boldsymbol{S}-\boldsymbol{I}): \Psi): \varepsilon^{\infty}, \text { in } \Omega
$$

$$
\text { ............................ }(10-a, b)
$$

where $\boldsymbol{I}$ is the fourth-order identity tensor, $\boldsymbol{S}$ Eshelby's tensor, and

$$
\boldsymbol{\Psi}=\left\{\boldsymbol{C}-\left(\boldsymbol{C}-\boldsymbol{C}^{\Omega}\right): \boldsymbol{S}\right\}^{-1}:\left(\boldsymbol{C}-\boldsymbol{C}^{\Omega}\right) \cdots \cdots(10-\mathrm{c})
$$

Since tensor $\boldsymbol{S}$ can be computed if the geometry of $\Omega$ is given, we write $S=S(\Omega ; C)$ and $\Psi=\Psi(\Omega ; C$, $\left.C^{\Omega}\right)$.

Although $\overline{\boldsymbol{C}}$ depend on the surface data, estimated are $\bar{C}$ when the linear displacements and the uniform tractions are prescribed; if the estimated $\overline{\boldsymbol{C}}$ under the two boundary conditions coincide, they might be a good representative of all possible overall moduli, since these two boundary conditions give the extreme value for $\langle e\rangle_{v}$ as shown by $(8-a, b)$. The three averaging schemes, the law of mixture (LM), the dilute distribution assumption (DD), and the self-consistent method (SC), can be summarized in a unified manner using (9) or (10) ; see Mura $^{12)}$ for detailed derivations. Under the linear displacements of $\varepsilon^{\circ}$, which yield $\langle\varepsilon\rangle_{V}=\varepsilon^{\circ}$ from (2), LM, DD, and SC approximate $\langle\varepsilon\rangle_{I}$ by $\varepsilon^{\circ},\left\{I+S^{I}: \overline{\Psi^{I}}\right\}: \overline{\varepsilon^{\circ}}$, and $\left\{\overline{I+} \boldsymbol{S}^{-I}: \Psi^{-I}\right\}: \varepsilon^{\circ}$, respectively, and estimate

$$
\begin{aligned}
\bar{C} & \simeq(1-f) \boldsymbol{C}^{M}+f \boldsymbol{C}^{I}, \\
\overline{\boldsymbol{C}} & \simeq \boldsymbol{C}^{M}+f\left(C^{I}-C^{M}\right):\left(I+S^{I}: \Psi^{I}\right), \\
\boldsymbol{C} & \simeq \boldsymbol{C}^{M}+f\left(C^{I}-C^{M}\right):\left\{I+\boldsymbol{S}^{-1}: \Psi^{-I}\right\} \\
& \ldots \ldots \ldots \ldots \ldots \ldots \ldots \cdots \cdots(11-\mathrm{a}, 12-\mathrm{a}, 13)
\end{aligned}
$$

where $S^{I}=\boldsymbol{S}\left(I ; C^{M}\right)$ and $\boldsymbol{\Psi}^{I}=\boldsymbol{\Psi}\left(I ; C^{M}, C^{I}\right)$, and $\bar{S}^{I}=S(I ; \bar{C})$ and $\bar{\Psi}^{I}=\Psi\left(I ; \bar{C}, C^{I}\right)$. Under the uniform tractions of $\sigma^{\circ}$, which yield $\langle\sigma\rangle_{V}=\sigma^{\circ}$ from (3), approximating $\langle\boldsymbol{\sigma}\rangle_{I}$ in the same manner as
$\langle\varepsilon\rangle_{I}, \underline{\mathrm{LM}}$ and $\underline{\mathrm{DD}}$ estimate

$$
\begin{aligned}
\boldsymbol{C} \simeq & \left\{(1-f)\left(\boldsymbol{C}^{M}\right)^{-1}+f\left(\boldsymbol{C}^{I}\right)^{-1}\right\}^{-1}, \\
\boldsymbol{C} \simeq & \left\{\left(\boldsymbol{C}^{M}\right)^{-1}+f\left(\left(\boldsymbol{C}^{I}\right)^{-1}-\left(\boldsymbol{C}^{M}\right)^{-1}\right)\right): \boldsymbol{C}^{M}: \\
& \left(I+\left(\boldsymbol{S}^{I}-\boldsymbol{I}\right): \boldsymbol{\Psi}^{I}:\left(\boldsymbol{C}^{M}\right)^{-1}\right\}^{-1} \cdots(11-\mathrm{b}, 12-\mathrm{b})
\end{aligned}
$$

but SC estimates (13). Estimates (12-a,b) and (13) are different. Nevertheless, they yield the same asymptotic behavior of $\bar{C}$ as $f$ goes to zero ; see Nemat-Nasser and Hori ${ }^{5)}$.

To clarify advantages and disadvantages of these averaging schemes, we set the following three requirements that are necessary (but not sufficient) for an ideal averaging scheme: I $\bar{C}$ for $f=0$ and 1 equal $\boldsymbol{C}^{M}$ and $\boldsymbol{C}^{\boldsymbol{I}}$, respectively ; II $\overline{\boldsymbol{C}}$ depend on the geometry and orientation of the inclusions ; III $\bar{C}$ are the same under the two different boundary conditions ( II means that a solid with, say, spherical inclusions does not behave in the same manner as one with, say, aligned long fibers, even though both have the same volume fraction, and III is necessary for the estimated $\overline{\boldsymbol{C}}$ to be a representative of all possible overall moduli). Table 1 shows whether an averaging scheme satisfies $(O)$ the requirements or not $(\times)$; FP is the finite part approximation which will be discussed in Sct. 5 . Although $\mathrm{SC}$ actually satisfies $\mathrm{I}$, it may yield an unrealistic estimate, say, $\overline{\boldsymbol{C}}=\boldsymbol{C}^{\boldsymbol{I}}$ for $f \neq 1$; see Hashin $^{13)}$.

\section{NEW AVERAGING SCHEME}

In a conventional modeling, $V$ is regarded as a finite body subjected to certain boundary conditions. However, it is possible to treat $V$ as a finite part in an infinite heterogeneous solid, $V^{\infty}$, which represents the material microstructure ; see Fig.2. Compared with a conventional one, this definition of $V$ is more realistic to relate how the microstructure is viewed macro- or micro-scopically. When $V^{\infty}$ is subjected to $\varepsilon^{\infty}$, field variables in the inside and outside of $V$ are not uniform, and simple surface data, such as linear displacements or uniform tractions, are not attained on $\partial V$. Under this condition, however, we can estimate average responses of $V$.

All exact equations $(9-\mathrm{a} \sim \mathrm{d})$ are still valid, even if $V$ is the finite part of the infinite solid. Moreover, the following relation is useful to consider the average field quantities over the finite part:for the infinite solid, $B^{\infty}$, introduced in Sct. 3, an average strain over an arbitrary ellipsoidal $\Omega^{\prime}$ which contains $\Omega$ within it is given by

$$
\langle\varepsilon\rangle_{\Omega^{\prime}}=\left(I+\frac{\Omega}{\Omega^{\prime}} S\left(\Omega^{\prime} ; C\right): \Psi\right): \varepsilon^{\infty}
$$

which is derived from the Tanaka-Mori theorem (Tanaka and Mori $^{14)}$ ). 
Table 1 Comparison of averaging schemes.

\begin{tabular}{llllll}
\hline \multirow{2}{*}{ requirement } & & LM & $\frac{\text { DD }}{\times}$ & $\frac{\text { SC }}{\times}$ & $\frac{\text { FP }}{\bigcirc}$ \\
& II & $\times$ & $\bigcirc$ & $\bigcirc$ & $\bigcirc$ \\
& III & $\times$ & $\times$ & $\bigcirc$ & $\bigcirc$ \\
\hline
\end{tabular}

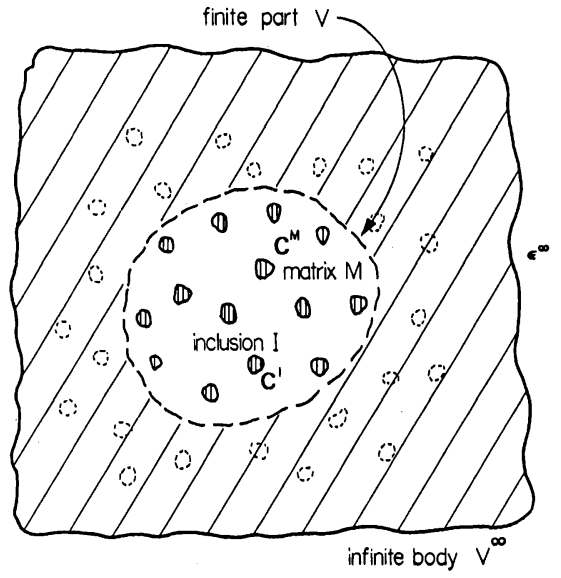

Fig.2 Finite part.
Whereas $\Omega$ corresponds to a single inclusion, $\Omega^{\prime}$ may correspond to the matrix surrounding the inclusion. If $\Omega^{\prime}$ and $\Omega$ are similar in shape and coaxial, (14) leads to $\langle\varepsilon\rangle_{\Omega^{\prime}-\Omega}=\varepsilon^{\infty}$. We may approximate the average strains and stresses over $M$ and $I$ by $\langle\varepsilon\rangle_{M} \simeq\langle\varepsilon\rangle_{\omega^{\prime}-\omega}=\varepsilon^{\infty}$ and $\langle\varepsilon\rangle_{I} \simeq\langle\varepsilon\rangle_{\Omega}=$ $\left(I+S^{I}: \Psi^{I}\right): \varepsilon^{\infty},\langle\boldsymbol{\sigma}\rangle_{M} \simeq\langle\boldsymbol{\sigma}\rangle_{\Omega^{\prime}-\Omega}=\boldsymbol{C}^{M}: \varepsilon^{\infty}$, and $\langle\boldsymbol{\sigma}\rangle_{I}$ $\simeq\langle\boldsymbol{\sigma}\rangle_{\Omega}=\boldsymbol{C}^{M}:\left\{\boldsymbol{I}+\left(\boldsymbol{S}^{I}-\boldsymbol{I}\right): \boldsymbol{\Psi}^{I}\right\}: \boldsymbol{\varepsilon}^{\infty}$. Hence, $\overline{\boldsymbol{C}}$ of $V$ are estimated by

$$
\bar{C} \simeq \boldsymbol{C}^{M}:\left\{I+f\left(\boldsymbol{S}^{I}-\boldsymbol{I}\right): \boldsymbol{\Psi}^{I}\right\}:\left(\boldsymbol{I}+f \boldsymbol{S}^{I}: \boldsymbol{\Psi}^{I}\right)^{-1}
$$

where $\langle\varepsilon\rangle_{V}$ and $\langle\boldsymbol{\sigma}\rangle_{V}$ are approximated by $\langle\varepsilon\rangle_{\Omega^{\prime}}=$ $\left\{\boldsymbol{I}+f \boldsymbol{S}^{I}: \boldsymbol{\Psi}^{I}\right\}^{-1}: \varepsilon^{\infty}$ and $\langle\boldsymbol{\sigma}\rangle_{\Omega^{\prime}}=\boldsymbol{C}^{M}:\left\{\boldsymbol{I}+f\left(\boldsymbol{S}^{I}\right.\right.$ $\left.-I): \Psi^{I}\right\}: \varepsilon^{\infty}$.

Benveniste ${ }^{8)}$ obtained (15) assuming that the inclusion should be subjected to $\langle\varepsilon\rangle_{M}$ rather than $\varepsilon^{\infty}$, while Mori and Wakashima ${ }^{9)}$ obtained (15) computing infinite summation of $\left(-f S^{I}: \Psi^{I}\right)^{n}$ or $\left(-f\left(\boldsymbol{S}^{I}-I\right): \Psi^{I}\right)^{n}$ from $n=0$ to $n=\infty$. Both regarded $V$ as a conventional finite body, and showed that the resulting $\bar{C}$ are the same under the linear displacements and the uniform tractions. However, boundary conditions on $\partial V$ are not required in the above derivations for the finite part, $V$. Note that (14) is exact whatever surface data on $\Omega^{\prime}$ are (Depending on relative location of $\Omega$ to $\Omega^{\prime}$, the surface data can vary, even though $B^{\infty}$ is subjected to the same $\varepsilon^{\infty}$ or $\boldsymbol{\sigma}^{\infty}$ ).

It is preferable to treat $M$ in a more statistical manner, since its geometry cannot be determined. To this end, we use the following identities for Eshelby's tensor:for any ellipsoidal $\Omega^{\prime}$ and for any isotropic $\boldsymbol{C}, \boldsymbol{S}\left(\Omega^{\prime}, \boldsymbol{C}\right)$ satisfies

$$
\begin{aligned}
& S_{i i j j}\left(\Omega^{\prime} ; \boldsymbol{C}\right)=S_{i i j j}(S ; \boldsymbol{C}), \\
& S_{i j i j}\left(\Omega^{\prime} ; \boldsymbol{C}\right)=S_{i j i j}(S ; C) \cdots \cdots \cdots \cdots \cdots(16-\mathrm{a}, \mathrm{b})
\end{aligned}
$$

where $\boldsymbol{S}$ denotes a sphere. Ellipsoidal geometry of
$\Omega^{\prime}$ can be specified by aspect ratios, $\rho_{2} \equiv a_{2} / a_{1}$ and $\rho_{3} \equiv a_{3} / a_{1}$, and angles, $\cos \phi_{1} \equiv \boldsymbol{e}_{3} \cdot \boldsymbol{b}_{1}$, and $\cos \theta_{1} \equiv$ $\boldsymbol{e}_{1} \cdot \boldsymbol{b}_{1}$, and $\cos \phi_{2} \equiv \boldsymbol{e}_{3} \cdot b_{2}$, of its major axes, where $a_{i}$ and $\boldsymbol{b}_{i}$ are the length and unit direction vector of the ith major axis with $\boldsymbol{e}_{i}$ being the unit vector in the $x_{i}$-direction ; see Fig.3. Then, (16-a) and (16-b) yields

$$
\begin{gathered}
\frac{1}{2 \pi^{3}} \int_{0}^{\pi} d \phi_{1} \int_{-\pi}^{\pi} d \theta_{1} \int_{\pi}^{0} d \phi_{2} \boldsymbol{S}^{\prime}\left(\rho_{2}, \rho_{3}, \phi_{1}, \theta_{1}, \phi_{2} ; \boldsymbol{C}\right) \\
\quad=\boldsymbol{S}(S ; \boldsymbol{C}) \ldots \ldots \ldots \ldots \ldots \ldots \ldots \ldots \ldots \ldots \ldots(16)
\end{gathered}
$$

for any $\rho_{2}$ and $\rho_{3}$. Identity (16-c) may suggest that a sphere $S$ of $\Omega / S=f$ is the most appropriate representation of $M$ in a statistical sense.

Now, a spherical finite part, $S$, is chosen for $\Omega^{\prime}$ in (14). First, following DD, we set $\boldsymbol{C}=\boldsymbol{C}^{M}$. The average field quantities over $V$ can be approximated by those over $S$, i.e., $\langle\varepsilon\rangle_{V} \simeq\langle\varepsilon\rangle_{S}=(I+$ $\left.f \boldsymbol{S}^{S}: \boldsymbol{\Psi}^{I}\right): \varepsilon^{\infty}$ and $\langle\boldsymbol{\sigma}\rangle_{V} \simeq\langle\boldsymbol{\sigma}\rangle_{S}=\left\{\boldsymbol{I}+f\left(\boldsymbol{S}^{S}-\boldsymbol{I}\right): \boldsymbol{\Psi}^{I}\right\}$ $: \varepsilon^{\infty}$, and hence, $\bar{C}$ are estimated by

$$
\bar{C} \simeq \boldsymbol{C}^{M}:\left\{\boldsymbol{I}+f\left(\boldsymbol{S}^{S}-\boldsymbol{I}\right): \boldsymbol{\Psi}^{I}\right\}:\left(\boldsymbol{I}+f \boldsymbol{S}^{S}: \boldsymbol{\Psi}^{I}\right)^{-1}
$$

where $\boldsymbol{S}^{S}=\boldsymbol{S}\left(S ; \boldsymbol{C}^{M}\right)$. Next, following SC, we set $\boldsymbol{C}$ $=\overline{\boldsymbol{C}}$, and render the average field quantities over $S$ $-\Omega$ and $\Omega$ to satisfy $\langle\boldsymbol{\sigma}\rangle_{S-\Omega}=\boldsymbol{C}^{S-\Omega}:\langle\boldsymbol{\varepsilon}\rangle_{S-\Omega}$ and $\langle\boldsymbol{\sigma}\rangle$ ${ }_{\Omega}=C^{\Omega}:\langle\varepsilon\rangle_{\Omega}$, which correspond to (9-c) and (9-d). To this end, fields due to another constant eigenstrain distributed in $B^{\Omega}, \varepsilon^{* \circ}$, are superposed onto Eshelby's solution; $\varepsilon^{*^{\circ}}$ produces uniform strain and stress fields satisfying $\sigma=C:\left(\varepsilon-\varepsilon^{* 0}\right)$. This superposition does not change the average strains due to $\varepsilon^{*}$, but subtracts $C: \varepsilon^{*^{\circ}}$ from the average stresses due to $\varepsilon^{*}$, i.e., $\langle\varepsilon\rangle_{S}=\varepsilon^{\infty}+(\Omega / S)$ $S^{s}: \varepsilon^{*},\langle\varepsilon\rangle_{\Omega} \simeq \varepsilon^{\infty}+S^{\Omega}: \varepsilon^{*},\langle\sigma\rangle_{S}=C:\left\{\varepsilon^{\infty}-\varepsilon^{*^{\circ}}+\right.$ $\left.(\Omega / S)\left(S^{S}-\boldsymbol{I}\right): \varepsilon^{*}\right\}$, and $\langle\boldsymbol{\sigma}\rangle_{\Omega}=\boldsymbol{C}:\left\{\varepsilon^{\infty}-\boldsymbol{\varepsilon}^{* \circ}+\left(\boldsymbol{S}^{\Omega}\right.\right.$ $\left.-I): \varepsilon^{*}\right\}$. These average field quantities over $S$ and $\Omega$ of $B^{\infty}$ are exact, and we can obtain $\varepsilon^{*}$ and $\varepsilon^{* \circ}$ to be 


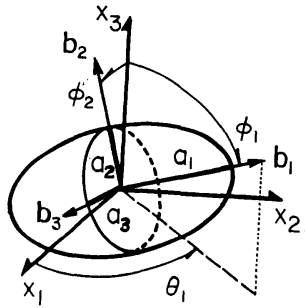

(a) Geometry of ellipsoid

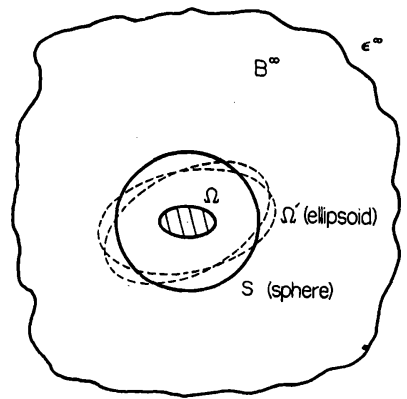

(b) Inclusion $\Omega$ within $S$ representing finite part $\Omega^{\prime}$

Fig.3

$\left.C: \varepsilon^{* \circ}=\left\{C-C^{\Omega}-\left(C-\left(C-C^{\Omega}\right): S^{\Omega}\right): \Phi\right)\right\}: \varepsilon^{\infty}$, $\varepsilon^{*}=\Phi: \varepsilon^{\infty} \ldots \ldots \ldots \ldots \ldots \ldots \ldots \ldots \ldots \ldots \ldots \ldots \ldots \ldots,(18-\mathrm{a}, \mathrm{b})$

where

$$
\begin{aligned}
& \Phi \equiv\left\{C-\left(C-C^{\Omega}\right): S^{\Omega}+\frac{(\Omega / S)}{1-(\Omega / S)}\left(C-C^{S-\Omega}\right):\right. \\
& \left.\left(\boldsymbol{S}^{S}-\boldsymbol{S}^{\Omega}\right)\right\}^{-1}:\left(\boldsymbol{C}^{S-\Omega}-\boldsymbol{C}^{\Omega}\right) \cdots \cdots \cdots \cdot(18-\mathrm{c})
\end{aligned}
$$

Like $\boldsymbol{S}$ or $\boldsymbol{\Psi}$, we write $\Phi=\Phi\left(\Omega / S ; S, \Omega ; \boldsymbol{C}, \boldsymbol{C}^{S-\Omega}\right.$, $C^{\Omega}$ ) with the first and second arguments denoting a volume fraction and geometry. From (18), $\langle\varepsilon\rangle_{S}$ and $\langle\boldsymbol{\sigma}\rangle_{S}$ can be computed, and $\overline{\boldsymbol{C}}$ are estimated by

$$
\begin{gathered}
\overline{\boldsymbol{C}} \simeq\left\{\boldsymbol{C}^{M}+f\left(\overline{\boldsymbol{C}}:\left(\overline{\boldsymbol{S}}^{s}-I\right)-\frac{1}{1-f}\left(\overline{\boldsymbol{C}}-\boldsymbol{C}^{M}\right):\right.\right. \\
\left.\left.\left(\overline{\boldsymbol{S}}^{S}-\overline{\boldsymbol{S}}^{I}\right)\right): \overline{\boldsymbol{\Phi}}\right\}:\left(\boldsymbol{I}+f \overline{\boldsymbol{S}}^{s}: \overline{\boldsymbol{\Phi}}\right)^{-1}
\end{gathered}
$$

where $\overline{\boldsymbol{S}}{ }^{S}=\boldsymbol{S}(S ; \overline{\boldsymbol{C}})$ and $\overline{\boldsymbol{\Phi}}=\boldsymbol{\Phi}\left(f ; S, I ; \overline{\boldsymbol{C}}, \boldsymbol{C}^{M}, C^{I}\right)$. We call estimates (17) and (19) the finite part approximation (FP).

\section{NEW AVERAGING SCHEME VS. CONVENTIONAL AVERAGING SCHEMES}

Now, we compare the new averaging scheme, FP, with the existing ones, LM, DD, and SC. Estimates (17) and (19) due to FP satisfy all the three requirements set in Sct. 3, although the three methods cannot ; (17) and (19) yield $\bar{C}=C^{M}$ for $f$ $=0$ and $\bar{C}=C^{I}$ for $f=1$ (I) ; they do not depend on the surface data on $\partial V$ (II) ; and they include effects of inclusion geometry through Eshelby's tensors (III). As shown in Table 1, FP appears more reasonable than the existing three methods. This is because FP uses exact relations among average field quantities over a finite part of an infinite domain, with the aid of the solutions of elasticity, (10) and (14). Hence, the conventional finite body definition may not be advantageous (In the author's opinion, the conventional definition may be made only to obtain another exact relation,
$\langle\varepsilon\rangle_{V}=\varepsilon^{\circ}$ or $\langle\boldsymbol{\sigma}\rangle_{V}=\boldsymbol{\sigma}^{\circ}$ when the linear displacements of $\varepsilon^{\circ}$ or the uniform tractions of $\boldsymbol{\sigma}^{\circ}$ are prescribed).

In Sct. 4, the average field quantities over $V$ are approximated by those over $S$, which represents all possible ellipsoidal $\Omega^{\prime}$ through integration (16-c). It might be expected (but cannot be proved at this moment) that similar integration of the right side of (5) vanishes, since it can take on a plus or minus value depending on relative location of $\Omega$ to $\Omega^{\prime}$. In a statistical sense, therefore, FP might render the overall moduli defined by $(7 \overline{-b})$ to coincide with those defined by (7-a).

According to the finite part concept, estimate (3.6) due to SC can be formulated if all tensors involved in (3.6) and (19) are isotropic. In this case, $S$ is replaced by an ellipsoidal domain $\Omega^{\prime}$ which is similar in shape and coaxial with $\Omega$, as in the case of deriving (15). Replacement of $S$ with $\Omega$ in (19) yields

$$
\overline{\boldsymbol{C}} \simeq\left\{\boldsymbol{C}^{M}+f \overline{\boldsymbol{C}}:\left(\overline{\boldsymbol{S}}^{I}-\boldsymbol{I}\right): \overline{\boldsymbol{\Psi}}^{\boldsymbol{T}}\right\}:\left(\boldsymbol{I}+f \overline{\boldsymbol{S}}^{I}: \overline{\boldsymbol{\Psi}}^{\prime}\right)^{-1}
$$

where $\overline{\Psi^{\prime \prime}}=\left\{\bar{C}-\left(\bar{C}-C^{I}\right): \bar{S}\right\}^{-1}:\left(C^{M}-C^{I}\right)=\bar{\Psi}$ : $\left(C^{I}-\bar{C}\right)^{-1}:\left(C^{M}-C^{I}\right)$. Multiply both sides of (20) with $\left(I+f \overline{\boldsymbol{S}^{I}}: \overline{\boldsymbol{\Psi}}^{\prime}\right)$ from the right and express $\overline{\boldsymbol{\Psi}}^{\prime \prime}$ in terms of $\bar{S}^{I}$, and we can rewrite (20) to be identical with (13), taking advantage of that isotropic tensors are comutable with each other. Hence, it is suggested that as geometry of the inclusion phase differs from a sphere, the estimate due to $\underline{\mathrm{SC}}$ (and (15)) becomes less accurate, since a sphere is the most appropriate geometry that represents all possible ellipsoids. As mentioned in Sct. 3, SC yields unrealistic estimates of the overall moduli when microinclusions are penny-shaped microcracks ; see Hashin ${ }^{13}$.

We compare FP with the Hashin-Shtrikman variational principle ( $\underline{\mathrm{HS}}$ ), which was originally proposed by Hashin and Shtrikman ${ }^{15), 16)}$; see Willis $^{6,7)}$ for neat generalization of the principle, 


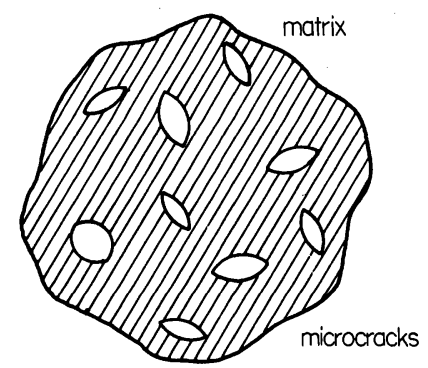

(a) Solid damaged by microcracks

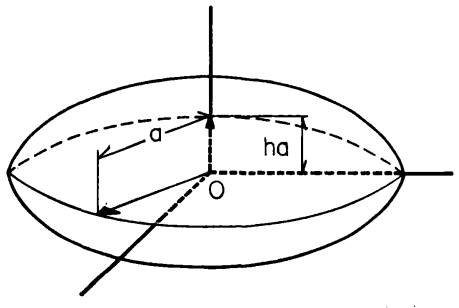

oblate spheroid

(b) Oblate spheroid

Fig.4

and Appendix presents a brief summary of HS (It should be noted that computation of bounds due to HS involves approximations and hence the bounds may not be exact, although the variational principle itself is mathematically correct without any underlying assumptions). From numerical computations, Mori and Wakashima" ${ }^{9}$ showed that their overall moduli, or FP with the assumption of $\boldsymbol{C}=\boldsymbol{C}^{M}$ and $\Omega^{\prime}=\Omega$, coincide with a bound due to $\underline{\mathrm{HS}}$. In fact, this coincidence can be proved analytically, as follows:for any, $\boldsymbol{C}$ Eshelby's tensor $\boldsymbol{S}$ satisfies $-\boldsymbol{I}$ $+\left\{I+S(\Omega ; C): C^{-1}:\left(C^{\Omega}-C\right)\right\}^{-1}=S: \Psi\left(C, C^{\Omega}, S\right.$ $(\Omega ; C))$. And a bound of the overall moduli due to $\underline{\mathrm{HS}}, \ddot{C}$, given by $\left(^{*}\right)$ of Appendix, can be rewritten as

$$
\ddot{C}=\boldsymbol{C}:\left\{\boldsymbol{I}+f\left(\boldsymbol{S}^{S}-\boldsymbol{I}\right): \Psi^{s}\right):\left(\boldsymbol{I}+f \boldsymbol{S}^{s}: \Psi^{s}\right)^{-1}
$$

where $\Psi^{\mathrm{s}}=\boldsymbol{\Psi}\left(\boldsymbol{C}, \boldsymbol{C}^{I} ; \boldsymbol{S}^{S}\right)$. If $\boldsymbol{C}$ is replaced by $\boldsymbol{C}^{M}$ and all inclusions are set to be spherical in the right side of (17), then, the resulting estimate becomes identical with (21).

Coincidence of (17) with (21) leads the following two remarks on FP:1) in HS of Willis's derivation, $S^{S}$ is determined from a statistical consideration of the interaction effects among all inhomogeneities. Hence, the coincidence supports the validity of using the fact that a sphere is the geometry that represents all possible ellipsoidal geometry in a sense that the Eshelby tensor for the sphere satisfies (16-c) ; and 2) as has been shown, the right side of (17) is the exact linear coefficients between $\langle\boldsymbol{\sigma}\rangle_{\Omega^{\prime}}$, and $\langle\varepsilon\rangle_{\Omega^{\prime}}$. For $\boldsymbol{C}=\boldsymbol{C}^{M}$, it can estimate the interaction between one inclusion and the surrounding matrix accurately. Hence, the coincidence suggests that interactions among adjacent inclusions always make a minus (or plus) contribution to the average strain energy of the material, since HS gives an upper (or lower) bound of the average strain energy ; see APPENDIX.

\section{EXAMPLE}

Overall moduli of a solid damaged by micro- cracks are studied as a simple example to demonstrate the effectiveness of FP. To reduce algebraic efforts to compute tensorial equations, we use the following expression for a fourth-order isotropic tensor, $\boldsymbol{A}$ :in terms of $\boldsymbol{E}^{1}$ and $\boldsymbol{E}^{2}$ which are defined by

$$
\begin{aligned}
& E_{i j k l}^{1} \equiv \frac{1}{3} \delta_{i j} \delta_{k l}, \\
& E_{i j k l}^{2} \equiv \frac{1}{2}\left(\delta_{i k} \delta_{j l}+\delta_{i l} \delta_{j k}\right)-\frac{1}{3} \delta_{i j} \delta_{k l} \cdots \cdots(22-\mathrm{a}, \mathrm{b})
\end{aligned}
$$

where $\delta_{i j}$ is Kronecker's delta, $\boldsymbol{A}$ is expressed by $\boldsymbol{A}$ $=A_{1} E^{1}+A_{2} E^{2}$. According to this expression, an isotropic elasticity tensor, $\boldsymbol{C}$, is given by $C_{1} \boldsymbol{E}^{1}+$ $C_{2} \boldsymbol{E}^{2}=3 K \boldsymbol{E}^{1}+2 \mu E^{2}$, where $K$ and $\mu$ are bulk and shear moduli. Since $E^{i}: E^{j}=\delta_{i j} \boldsymbol{E}^{j}$ ( $j$ not summed), a tensorial equation for isotropic tensors can be transformed into the corresponding two scalar equations for the coefficients with respect to $\boldsymbol{E}^{1}$ and $\boldsymbol{E}^{2}$; see, for example, Hill ${ }^{4)}$ or Budiansky ${ }^{177}$.

In the case that a solid is damaged by randomly distributed microcracks, as shown in Fig.4 (a), the resulting overall moduli become isotropic ; see Sahasakmontri et $a l .^{18)}$ for a concise literature survey of this problem. For simplicity, a microcrack, $C$, is assumed to be penny-shaped of radius $a$ with traction free surfaces, and we regard $C$ as the limit of an oblate spheroidal cavity, $\mathrm{O}$, of radius $a$ and thickness $a h(h<1)$, as its thickness vanishes ; see Fig.4 (b). As $h$ goes to zero, the corresponding $\Psi=\Psi(O ; C, 0)$ diverges as fast as $1 / h$, but the volume of $C$ becomes zero as fast as $h$. Hence, $\langle\varepsilon\rangle_{0}$ remains finite. Indeed, defining $\boldsymbol{r}$ from $\boldsymbol{\Psi}(\mathrm{O} ; \boldsymbol{C}, \mathbf{0})$ by

$$
\boldsymbol{r}=\boldsymbol{r}\left(\boldsymbol{C} ; \boldsymbol{S}^{\mathrm{O}}\right)=\lim _{h \rightarrow 0} h \boldsymbol{\Psi}(\mathrm{O} ; \boldsymbol{C}, \mathbf{0})=\lim _{h \rightarrow 0} h\left(\boldsymbol{I}-\boldsymbol{S}^{0}\right)^{-1}
$$

with $\boldsymbol{S}^{0}=\boldsymbol{S}(0 ; \boldsymbol{C})$, we can obtain $\langle\boldsymbol{\varepsilon}\rangle_{C}$ as the limit of $\langle\varepsilon\rangle_{0}$, i.e., $r: \varepsilon^{\infty}$. It is assumed that the average strain over the crack phase consisting of a random distribution of microcracks can be given by taking sum of $\langle\varepsilon\rangle_{c}$ for cracks of various sizes and orientations. Such summation results in relating the 
average strain over the crack phase to $\varepsilon^{\infty}$ through a certain isotropic tensor. This tensor, $\boldsymbol{R}$, can be constructed from $r$ through $R_{i i j j}=r_{i i j j}$ and $R_{i j i j}=$ $r_{i j i j}$, which yield

$$
\begin{aligned}
\boldsymbol{R} & =\boldsymbol{R}\left(\boldsymbol{C} ; \boldsymbol{S}^{0}\right)=R_{1} \boldsymbol{E}^{1}+R_{2} \boldsymbol{E}^{2} \\
& =\frac{1}{\pi} \frac{4\left(1-\nu^{2}\right)}{3(1-2 \nu)} \boldsymbol{E}^{1}+\frac{1}{\pi} \frac{8(1-\nu)(5-\nu)}{15(2-\nu)} \boldsymbol{E}^{2}
\end{aligned}
$$

where $\nu$ is the Poisson ratio of $C$, given by ( $3 K-2$ $\mu) /(6 K+2 \mu)$.

Using the above results, we substitute (24) into (12-a), (12-b), or (13), respectively, and estimate $\bar{C}$ due to $\underline{D D}$ and $\mathrm{SC}$ by

$$
\begin{aligned}
& \overline{\boldsymbol{C}} \simeq \boldsymbol{C}^{M}:\left(\boldsymbol{I}-d \boldsymbol{R}^{M}\right), \quad \overline{\boldsymbol{C}} \simeq \boldsymbol{C}^{M}:\left(\boldsymbol{I}+d \boldsymbol{R}^{M}\right)^{-1}, \\
& \overline{\boldsymbol{C}} \simeq \boldsymbol{C}^{M}:(\boldsymbol{I}-d \overline{\boldsymbol{R}}) \cdots \cdots \cdots \cdots \cdots \cdots(25-\mathrm{a}, \mathrm{b}, 26)
\end{aligned}
$$

where $\boldsymbol{R}^{M}=\boldsymbol{R}\left(\boldsymbol{C}^{M} ; \boldsymbol{S}\left(\mathrm{O} ; \boldsymbol{C}^{M}\right)\right)$ and $\overline{\boldsymbol{R}}=\boldsymbol{R}(\overline{\boldsymbol{C}} ; S$ $(\mathrm{O} ; \overline{\boldsymbol{C}}))$, and $\boldsymbol{d}$ is the crack density defined by $1 / V \sum 4 \pi a_{\alpha}^{3} / 3$ with summation being taken for all cracks of radius $a_{\alpha}$ in $V$; see Budiansky and $\mathrm{O}^{\prime}$ Connell ${ }^{19)}$ or Horii and Nemat-Nasser ${ }^{20)}$. It should be noted that LM cannot be used to the solid damaged by cracks, since the volume fraction of cracks is zero.

We apply (23) and (24) to FP, and compare the above estimates due to $\underline{\mathrm{DD}}$ and $\underline{\mathrm{SC}}$. First, according to (17), we consider a penny-shaped crack, $C$, in $B^{\infty}$ with elasticity $C=C^{M}$, and choose a spherical region, $S$, such that it contains $C$ within it. In terms of $\boldsymbol{r}$, the average field quantities over $S$, $\langle\varepsilon\rangle_{s}$ and $\langle\boldsymbol{\sigma}\rangle_{s}$, can be computed exactly for this isolated crack. Summation of $\langle\boldsymbol{\varepsilon}\rangle_{S}$ and $\langle\boldsymbol{\sigma}\rangle_{S}$ for cracks of various sizes and orientations yields $\Sigma$ $\langle\boldsymbol{\varepsilon}\rangle_{s}=\left\{\boldsymbol{I}+\left(4 \pi a^{3} / 3 S\right) \boldsymbol{S}^{S}: R^{M}\right\}: \varepsilon^{\infty}$ and $\sum\langle\boldsymbol{\sigma}\rangle_{S}=$ $\boldsymbol{C}^{M}:\left\{\boldsymbol{I}+\left(4 \pi a^{3} / 3 S\right)\left(\boldsymbol{S}^{S}-\boldsymbol{I}\right): \boldsymbol{R}^{M}\right\}: \varepsilon^{\infty}$. As is seen, these sums are related to $\varepsilon^{\infty}$ through an isotropic tensor. Approximation of $\langle\boldsymbol{\varepsilon}\rangle_{V} \simeq \sum\langle\boldsymbol{\varepsilon}\rangle_{S}$ and $\langle\boldsymbol{\sigma}\rangle_{V}$ $\simeq \sum\langle\boldsymbol{\sigma}\rangle_{s}$ yields

$$
\bar{C} \simeq C^{M}:\left\{\boldsymbol{I}+d\left(S^{S}-\boldsymbol{I}\right): \boldsymbol{R}^{M}\right\}:\left\{\boldsymbol{I}+d \boldsymbol{S}^{S}: \boldsymbol{R}^{M}\right)^{-1}
$$

Next, according to (19), we replace elasticity of $B^{\infty}, C$, from $C^{M}$ to $\bar{C}$. In this setting, two eigenstrains must be considered as explained in Sct. 5. The limiting procedure deriving (23) is applied to obtain these eigenstrains, as follows:let two constant eigenstrains, $\varepsilon^{* \circ}$ and $\varepsilon^{*}$, be distributed in $B^{\infty}$ and an oblate spheroid, $\mathrm{O}$, respectively. As the thickness of $\mathrm{O}$ vanishes and $\mathrm{O}$ approaches a crack $C$, the following exact relation hold for the averaging field quantities over $S$ and $C$ :

$$
\begin{aligned}
& \langle\varepsilon\rangle_{S}=\varepsilon^{\infty}+\frac{1}{S} \frac{4}{3} \pi a^{3} \lim _{h \rightarrow 0}\left(S^{S}-S^{0}\right):\left(h \varepsilon^{*}\right), \\
& \langle\varepsilon\rangle_{C}=\varepsilon^{\infty}+\lim _{h \rightarrow 0} S^{0}: \varepsilon^{*}
\end{aligned}
$$

$$
\begin{gathered}
\langle\boldsymbol{\sigma}\rangle_{S}=\boldsymbol{C}:\left\{\boldsymbol{\varepsilon}^{\infty}-\boldsymbol{\varepsilon}^{* \circ}+\frac{1}{S} \frac{4}{3} \pi a^{3} \lim _{h \rightarrow 0}\left(\boldsymbol{S}^{S}-\boldsymbol{I}\right):\right. \\
\left.\left(h \boldsymbol{\varepsilon}^{*}\right)\right\}, \\
\langle\boldsymbol{\sigma}\rangle_{C}=\boldsymbol{C}:\left\{\boldsymbol{\varepsilon}^{\infty}-\boldsymbol{\varepsilon}^{*^{\circ}}+\lim _{h \rightarrow 0}\left(\boldsymbol{S}^{0}-\boldsymbol{I}\right): \boldsymbol{\varepsilon}^{*}\right\}
\end{gathered}
$$

Provided that the average field relations, $\langle\boldsymbol{\sigma}\rangle_{s-C}=$ $C^{\mathrm{M}}:\langle\varepsilon\rangle_{S-C}$ and $\langle\boldsymbol{\sigma}\rangle_{C}=\mathbf{0}$, are enforced, $\boldsymbol{\varepsilon}^{* \circ}$ and $\varepsilon^{*}$ are obtained to be

$$
\begin{aligned}
& \varepsilon^{* \circ}=\varepsilon^{\infty}+\lim _{h \rightarrow 0}\left(\boldsymbol{S}^{0}-\boldsymbol{I}\right): \varepsilon^{*}, \quad \lim _{h \rightarrow 0} h \varepsilon^{*}=\boldsymbol{r}^{\prime}: \varepsilon^{\infty} \\
& \text { (2............................. b }(29)
\end{aligned}
$$

where

$$
\begin{aligned}
\boldsymbol{r}^{\prime}=\lim _{h \rightarrow 0} h\left\{\boldsymbol{I}-\boldsymbol{S}^{\mathrm{o}}+\frac{4}{3} \pi a^{3} h\left(\boldsymbol{I}-\boldsymbol{C}^{-1}: \boldsymbol{C}^{M}\right):\right. \\
\left.\left(\boldsymbol{S}^{S}-\boldsymbol{S}^{\mathbf{0}}\right)\right\}^{-1}: \boldsymbol{C}^{-1}: \boldsymbol{C}^{M} \cdots \cdots \cdots(29-\mathrm{c})
\end{aligned}
$$

From $\boldsymbol{r}^{\prime}$, an isotropic tensor, $\boldsymbol{R}^{\prime}$, can be constructed in the same manner as $\boldsymbol{R}$ from $\boldsymbol{r}$. Since $\boldsymbol{r}^{\prime}$ is written as $\boldsymbol{r}^{\prime}=\boldsymbol{r}^{\prime}\left(\boldsymbol{C}, \boldsymbol{C}^{M} ; \boldsymbol{S}^{S}, \boldsymbol{S}^{0}\right)$, we write $\boldsymbol{R}^{\prime}=\boldsymbol{R}^{\prime}$ $\left(C, C^{M} ; S^{S}, S^{0}\right)$. The average strain and stress over $V$ are approximated by $\langle\varepsilon\rangle_{V} \simeq\langle\varepsilon\rangle_{s}=\left(\boldsymbol{I}+d \overline{\boldsymbol{S}}^{s}\right.$ : $\left.\overline{\boldsymbol{R}}^{\prime}\right): \varepsilon^{\infty}$ and $\langle\boldsymbol{\sigma}\rangle_{V} \simeq\langle\boldsymbol{\sigma}\rangle_{S}=\boldsymbol{C}^{M}:\left\{\boldsymbol{I}+d\left(\bar{S}^{s}-\boldsymbol{I}\right): \overline{\boldsymbol{R}}^{\prime}\right\}$ $: \varepsilon^{\infty}$, and hence $\bar{C}$ are estimated by

$$
\overline{\boldsymbol{C}} \simeq \boldsymbol{C}^{M}:\left\{\boldsymbol{I}+d\left(\overline{\boldsymbol{S}}^{s}-\boldsymbol{I}\right): \overline{\boldsymbol{R}}^{\prime}\right\}:\left(\boldsymbol{I}+d \overline{\boldsymbol{S}}^{s}: \overline{\boldsymbol{R}}^{\prime}\right)^{-1}
$$

where $\overline{\boldsymbol{R}}^{\prime}=\boldsymbol{R}^{\prime}\left(\overline{\boldsymbol{C}}, \boldsymbol{C}^{M} ; \overline{\boldsymbol{S}}^{s}, \overline{\boldsymbol{S}}^{\mathrm{o}}\right)$ with $\overline{\boldsymbol{S}}^{\mathrm{o}}=\boldsymbol{S}(\mathrm{O} ; \overline{\boldsymbol{C}})$

Fig.5 shows the variation of the overall moduli estimated by DD, SC, and FP, ((25), (26), (27) and (30)) with respect to the crack density $d$; in terms of the overall bulk and shear moduli, $\bar{K}$ and $\bar{\mu}, \bar{C}$ are expressed by $3 \bar{K} \boldsymbol{E}^{1}+2 \bar{\mu} \boldsymbol{E}^{2}$, and the ratio of these moduli to the corresponding one of the matrix, i.e., $\bar{C}_{1} / C_{1}^{M}=\bar{K} / K^{M}$ and $\bar{C}_{2} / C_{2}^{M}=\bar{\mu} / \mu^{M}$, is plotted, with the matrix Poisson ratio, $\nu^{M}$, being 0.3 . Both $\bar{K}$ and $\bar{\mu}$ can be easily computed from the scalar equations that are directly reduced from the tensorial equation for $\overline{\boldsymbol{C}}$, as isotropic tensors are expressed in terms of $\boldsymbol{E}^{1}$ and $\boldsymbol{E}^{2}$ defined by (22). Fig. 5 shows that $\bar{K}$ and $\bar{\mu}$ estimated by $\underline{D D}$ and $\mathrm{SC}$ vanish at a certain crack density. As explained in Sct. 5, we can view SC as FP with an assumption of taking a finite part surrounding a crack to be similar in shape to it and setting $\boldsymbol{C}=\overline{\boldsymbol{C}}$. Such a finite part may cause the unrealistic estimates of $\vec{C}$ due to $\underline{\mathrm{SC}}$. The theoretical predictions due to FP need to be compared with accurate experimental data, in order to clarify the effectiveness of this new averaging scheme in a more transparent manner. However, it seems that the results presented suggest that the proposed FP can make more reasonable estimates of the overall moduli, at least compared with those due to the conventional averaging schemes. 


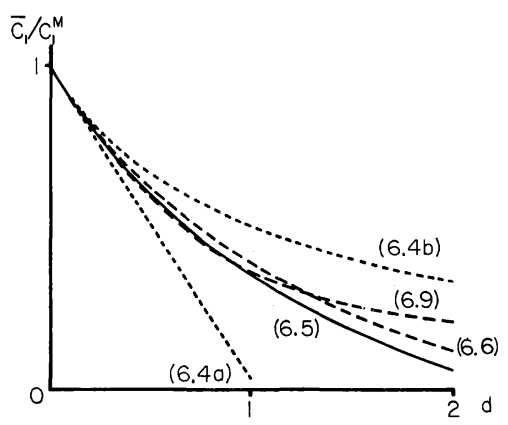

(a) Variation of $\bar{C}_{1}$ with respect to $d$

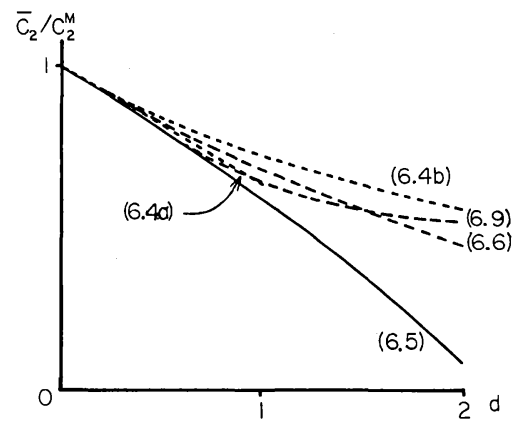

(b) Variation of $\bar{C}_{2}$ with respect to $d$

Fig.5

\section{APPENDIX:HASHIN-SHTRIKMAN VARIATIONAL PRINCIPLE}

According to Willis ${ }^{6,7)}$, the Hashin-Shtrikman variational principle is formulated as follows:suppose that a finite body $V$ with heterogeneous elasticity $C^{\prime}$ is subjected to the linear displacements $\boldsymbol{x} \cdot \boldsymbol{\varepsilon}^{\circ}$. Instead of $V$, we consider an equivalent homogeneous solid $V^{*}$ with arbitrary uniform elasticity $C$ and eigenstrains $e^{*}$. Define a functional $I$ for $e^{*}$ by

$$
\begin{aligned}
I \equiv & \frac{1}{2}\left\langle\left(-C: e^{*}\right):\left\{\left(C^{\prime}-C\right)^{-1}:\left(-C: e^{*}\right)\right.\right. \\
& \left.\left.-\varepsilon-2 \varepsilon^{\circ}\right\}\right\rangle_{V^{*}}
\end{aligned}
$$

where $\varepsilon$ is the strain field produced by $e^{*}$, satisfying zero displacements on $\partial V^{*}$. From

$$
\begin{aligned}
\delta I= & \left\langle\left(-C: \delta e^{*}\right):\left\{\left(C^{\prime}-C\right)^{-1}:\left(-C: e^{*}\right)\right.\right. \\
& \left.\left.-\varepsilon-\varepsilon^{\circ}\right\}\right\rangle_{V^{*}}
\end{aligned}
$$

it is seen that the Euler equation of $I$ is equivalent with $\sigma=C^{\prime}:\left(\varepsilon+\varepsilon^{\circ}\right)$. Hence, $\varepsilon^{*}$ satisfying $\partial I=0$ produces the same field variables in $V^{*}$ as those in $V$, and $I\left(\varepsilon^{*}\right)=\varepsilon^{\circ}:(C-\bar{C}): \varepsilon^{\circ} / 2$. Furthermore, if $C^{\prime}$ $-C$ is positive- (or negative-) definite, the following inequality holds for any $e^{*}$ :

$$
I\left(\varepsilon^{*}\right) \geqq(\text { or } \leqq) I\left(e^{*}\right)
$$

With the aid of Eshelby's solution, the value of $I$ can be estimated. Consider a general multi-phase material. Let $I^{\alpha}$ be the $\alpha$ th inclusion phase of elasticity $\boldsymbol{C}^{\alpha}$ and the volume fraction $f^{\alpha}$. For a piecewise constant distribution of $\boldsymbol{e}^{*}$ which takes $\left\langle\boldsymbol{e}^{*}\right\rangle_{\alpha}$ in $I^{\alpha}$,

$$
\begin{aligned}
I \simeq & \frac{1}{2} \sum_{\alpha} f^{\alpha}\left\langle\boldsymbol{e}^{*}\right\rangle_{\alpha}: \boldsymbol{C}:\left\{\left(\boldsymbol{C}^{\alpha}-\boldsymbol{C}\right)^{-1}+\boldsymbol{S}^{S}: \boldsymbol{C}^{-1}\right\}: \\
& \left(\boldsymbol{C}:\left\langle\boldsymbol{e}^{*}\right\rangle_{\alpha}\right)-\frac{1}{2} \sum_{\alpha} \sum_{\beta} f^{\alpha} f^{\beta}\left\langle\boldsymbol{e}^{*}\right\rangle_{\alpha}: \boldsymbol{C}: \boldsymbol{S}^{S}:\left\langle\boldsymbol{e}^{*}\right\rangle_{\beta} \\
& -\sum_{\alpha} f^{\alpha}\left\langle\boldsymbol{e}^{*}\right\rangle_{\alpha}: \varepsilon^{\circ}
\end{aligned}
$$

where $\boldsymbol{S}^{S}=\boldsymbol{S}(S ; \boldsymbol{C})$ with $S$ being a sphere. The above quadratic form can be extremized, and a bound for $\bar{C}$ is given by $\varepsilon^{\circ}: \bar{C}: \varepsilon^{\circ} / 2 \geqq$ (or $\left.\leqq\right)$ $\varepsilon^{\circ}: \mathcal{C}: \varepsilon^{\circ} / 2$, where

$$
\begin{aligned}
& \stackrel{*}{\boldsymbol{C}} \equiv\left\{\sum_{\alpha} f^{\alpha} \boldsymbol{C}^{\alpha}:\left\{\boldsymbol{I}+\left(S^{S}-\boldsymbol{I}\right): \boldsymbol{C}^{-1}:\left(\boldsymbol{C}^{\alpha}-\boldsymbol{C}\right)\right\}^{-1}\right\}: \\
& \left\{\sum_{\beta} f^{\beta} \boldsymbol{C}\left\{\boldsymbol{I}+\boldsymbol{S}^{s}: \boldsymbol{C}^{-1}:\left(\boldsymbol{C}^{\beta}-\boldsymbol{C}\right)\right\}^{-1}\right\}^{-1} \cdots \cdots(
\end{aligned}
$$

\section{REFERENCES}

1) Christensen, R.M.:Mechanics of Composite Materials, John Wiley \& Sons, New York, 1979.

2) Hashin, Z.:Analysis of composite materials - a survey, J. App. Mech., Vol.50, pp.481 505, 1983.

3) Introduction to Mathematical Models of Material Characteristics (edited by Kuranishi, S.), JSCE, 1989.

4) Hill, R.:The elastic behavior of a crystalline aggregate, Proc. Phys. Soc., A 65, pp.349 534, 1952.

5) Nemat-Nasser, S. and Hori, M.:Elastic solids with microdefects, in Micromechanics and Inhomogeneity (edited by Weng, G.J., Taya, M. and Abé, H.), pp.297 $320,1989$.

6) Willis, J.R.:Bounds and self-consistent estimates for the. overall properties of anisotropic composites, J. Mech. Phys. Solids, Vol.25, pp.185 202, 1977.

7) Willis, J.R.:Variational and related methods for the overall properties of composites, Adv. App. Mech., Vol.21 (edited by Yih, C-S.), pp.1 78, Academic Press, New York, 1981.

8) Benveniste, Y.:Mori-Tanaka's theory in composites, Mech. of Mat., Vol.6, pp.147 157, 1987.

9) Mori, T. and Wakashima, K.:Successive iteration method in the evaluation of average fields in elastically inhomogeneous materials, in Micromechanics and Inhomogeneity (edited by Weng, G.J., Taya, M. and Abé, H.), pp.269 282, 1989.

10) Eshelby, J.D.:The determination of the elastic field of an ellipsoidal inclusion, and related problems, Proc. Roy. Soc., A 241, pp.376 396, 1957.

11) Eshelby, J.D.:Elastic inclusions and inhomogeneities, in Progress in Solid Mechanics 2 (edited by Sneddon, I.N. and Hill, R.), pp.89 140, North-Holland, Amsterdam, 1961. 
12) Mura, T.:Micromechanics of Defects in Solids, Martinus Nijhoff, New York, 1982.

13) Hashin, Z.:The differential scheme and its application to cracked materials, J. Mech. Phys. Solids, Vol.36, pp.719 734, 1988.

14) Tanaka, K. and Mori, T.:Note on volume integrals of the elastic field around an ellipsoidal inclusion, J. Elasticity, Vol.2, pp.199 200, 1972.

15) Hashin, Z. and Shtrikman, S.:On some variational principles in anisotropic and nonhomogeneous and elasticity, J. Mech. Phys. Solids, Vol.10, pp.335 342, 1962.

16) Hashin, Z. and Shtrikman, S.:A variational approach to the theory of the elastic behavior of polycrystals, J. Mech. Phys. Solids, Vol.10, pp.343 352, 1962.
17) Budiansky, B.:On the elastic moduli of some heterogeneous solids, J. Mech. Phys. Solids, Vol.13, pp.223 227, 1965.

18) Sahasakmontri, K., Horii, H., Hasegawa, A. and Nishino, F.:Mechanical properties of solids containing a doubly periodic rectangular array of cracks, Structural Eng./Earthquake Eng., Vol.4, pp.125 135, 1987.

19) Budiansky, B. and O'Connell, R.J.:Elastic moduli of a cracked solid, Int. J. Solids, Structures, Vol.12, pp.81 97, 1976.

20) Horii, H. and Nemat-Nasser, S.:Overall moduli of solids with microcracks:load-induced anisotropy, J. Mech. Phys. Solids, Vol.31, pp.163 171, 1983.

(Received March 24, 1990)

\section{不均一弾性体の平均弾性}

$$
\text { 堀 宗朗・三浦 尚 }
$$

不均一な材料の全体的な挙動を推定することは,工学分野全般での重要な問題である. 本論文では, 物理場の体積平均に成立する関係式を用いて不均一材料の平均挙動を評価 することから, 平均弾性の推定のための有限体近似法を提案する. この方法は既存の推 定法の欠点を克服している. 亀裂による損傷を被った材料を例として, 提案され有限体 近似法と既存の推定法が予測する平均弾性の比較を行った. 